\title{
Compliance trumps oxygenation: Predicting quality with ex vivo lung perfusion
}

\author{
Vishnu Vasanthan, BSc, and Jayan Nagendran, MD, PhD, FRCSC
}

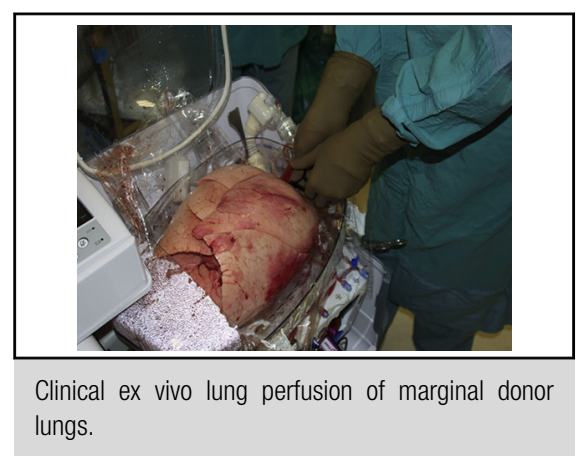

Central Message

Trends in lung compliance are more predictive of donor lung quality than measures of oxygenation during ex vivo lung perfusion.

See Article page 1376.
Mortality rates continue to be unacceptably high for potential recipients awaiting lung transplantation. Ex vivo lung perfusion (EVLP) is an effective modality to both evaluate and recondition donor lungs. Despite the strengths of EVLP, there are no consistent guidelines by which decisions can be based to determine the viability of donor lungs for transplantation. Surgical judgment of mixed physiologic measurements currently determines donor lung suitability. Clearly, a defined algorithm of EVLP lung function is required to allow for adoption of the EVLP technique by more lung transplant centers.

In this issue of the Journal, Sanchez and colleagues ${ }^{1}$ provide a simple and elegant EVLP algorithm for determining donor lung suitability. They focus on compliance over oxygenation (pulmonary arterial oxygen tension-left atrial oxygen tension) in assessing lung function, appreciating that oxygenation can appear spuriously elevated despite deteriorating lung quality. ${ }^{2}$ Preclinical studies from the Toronto group ${ }^{3}$ have also shown that oxygenation alone does not predict donor lung function after transplantation, and parameters, including compliance, also decrease during EVLP of injured lungs. Interestingly pulmonary vascular resistance and peak airway pressures were similar between suitable and nonsuitable lungs. It is possible that these other physiologic parameters would deteriorate over longer EVLP runs, and is not appreciated in the first 3 hours as observed by Sanchez and colleagues. ${ }^{1}$ By definition of static compliance, with plateau airway pressures and positive end expiratory pressures not changing significantly, there is a decrease in tidal volumes of the lungs deemed not suitable, despite sometimes having adequate oxygenation of the aceullar perfusate. Identifying poor or decreasing compliance as an early indicator of lung dysfunction is a signal to alter perfusion strategies, including therapeutic bronchoscopy and administration of nebulized bronchodilators, as is practiced at our center. Allowing a further hour of perfusion to track the trend toward declining function may also represent time in which reconditioning and reparative strategies may be employed.

The current evidence for the efficacy of EVLP grows with results from the Toronto group, ${ }^{4}$ the TransMedicssponsored International Randomized Study of the TransMedics Organ Care System (OCS Lung) for Lung Preservation and Transplantation (INSPIRE Trial), ${ }^{5,6}$ and the Xvivo-sponsored Normothermic Ex Vivo Lung Perfusion as an Assessment of Marginal Donor Lungs trial, and points toward wider use of EVLP strategies to increase donor lung use rates. Thoughtful consideration to the value of EVLP-derived physiologic parameters is critical to avoid harm or damaging potentially acceptable lungs with EVLP. These parameters can also help grow the use of donation after circulatory death lung donors, because EVLP has been successful in expanding the donor pool to safely to include this type of donor. ${ }^{8,9}$ This brief report provides important corroborating evidence to the EVLP literature because it validates the findings of preclinical studies and decreases the reliance on judgment with focus on objective data to determine donor lung viability. Knowledge gained from the data collected by Sanchez and colleagues ${ }^{1}$ may also help explain why current clinical EVLP programs perfuse lungs for only 4 to 6 hours, ${ }^{4-7}$ yet most preclinical studies show great stability on EVLP platforms for $>12$ hours. There is also an increasing volume of novel reparative approaches using EVLP for donor-specific ${ }^{10}$ and recipientspecifc $^{11}$ conditions, which may further extend the use of 
EVLP for assessment and organ-specific repair. The lofty goals of EVLP include introduction of cell and gene therapies. For more complex therapeutic strategies to evolve, clinical EVLP protocols must be extended safely for $>12$ hours to allow for delivery of these therapies and assessment of the intervention. As such, evidence for the significance of compliance during EVLP assessment, in correlation with oxygenation, appreciating that oxygenation is likely to follow trends in compliance, is an important and insightful step forward.

\section{References}

1. Sanchez PG, Rajagopal K, Pham SM, Griffith BP. Defining quality during ex vivo lung perfusion: The University of Maryland experience. J Thorac Cardiovasc Surg. 2015:150:1376-7.

2. Lowe K, Alvarez DF, King JA, Stevens T. Perivascular fluid cuffs decrease lung compliance by increasing tissue resistance. Crit Care Med. 2010;38:1458-66.

3. Yeung JC, Cypel M, Machuca TN, Koike T, Cook DJ, Bonato R, et al. Physiologic assessment of the ev vivo donor lung for transplantation. $J$ Heart Lung Transplant. 2011;31:1120-6.

4. Cypel M, Yeung JC, Liu M, Anraku M, Chen F, Karolak W, et al. Normothermic ex vivo lung perfusion in clinical lung transplantation. $N$ Engl J Med. 2011;364: $1431-40$.
5. Warnecke G, Weigmann B, Van Raemdonck D, Massard G, Santelmo N, Falcoz P, et al. The INSPIRE international lung trial with the Organ Care System Technology (OCS ${ }^{\mathrm{TM}}$ ). J Heart Lung Transplant. 2013;32(Suppl):S16.

6. Warnecke G, Moradiellos J, Tudorache I, Kühn C, Avsar M, Wiegmann B, et al. Normothermic perfusion of donor lungs for preservation and assessment with the Organ Care System Lung before bilateral transplantation: A pilot study of $12 \mathrm{pa}-$ tients. Lancet. 2012;380:1851-8.

7. Sanchez P, Davis R, D'ovidio F, Weyan M, Camp P, Cantu E III, et al. Normothermic Ex Vivo Lung Perfusion as an Assessment of Marginal Donor Lungs-the NOVEL lung trial. J Heart Lung Transplant. 2013;32: 1053-2498.

8. Bozso S, Vasanthan V, Luc JG, Kinaschuk K, Freed D, Nagendran J. Lung transplantation from donors after circulatory death using portable ex vivo lung perfusion. Can Respir J. 2014;22:50-9.

9. Machuca TN, Mercier O, Collaud S, Tikkanen J, Krueger T, Yeung JC, et al. Lung transplantation with donation after circulatory determination of death donors and the impact of ex vivo lung perfusion. Am J Transplant. 2015;15: 993-1002.

10. Luc JG, Bozso SJ, Freed DH, Nagendran J. Successful repair of donation after circulatory death lungs with large pulmonary embolus using the lung organ care system for ex vivo thrombolysis and subsequent clinical transplantation. Transplantation. 2015;99:e1-2.

11. Ceulemans LJ, Monbaliu D, Verslype C, van der Merwe S, Laleman W, Vos R, et al. Combined liver and lung transplantation with extended normothermic lung preservation in a patient with end-stage emphysema complicated by drug-induced acute liver failure. Am J Transplant. 2014; 14:2412-6. 